\title{
Questes
}

\section{Sans naissance, pas de mariage ? Le procès en nullité du mariage de Louis XII et Jeanne de France (1498)}

\section{Stéphanie Richard}

\section{OpenEdition}

\section{Journals}

Édition électronique

URL : http://journals.openedition.org/questes/761

DOI : 10.4000/questes.761

ISSN : 2109-9472

Éditeur

Les Amis de Questes

\section{Édition imprimée}

Date de publication : 15 janvier 2014

Pagination : 47-66

ISSN : 2102-7188

\section{Référence électronique}

Stéphanie Richard, « Sans naissance, pas de mariage ? Le procès en nullité du mariage de Louis XII et Jeanne de France (1498) », Questes [En ligne], 27 | 2014, mis en ligne le 15 janvier 2014, consulté le 01 mai 2019. URL : http://journals.openedition.org/questes/761 ; DOI : 10.4000/questes.761

\section{(c) Association des amis de "Questes »}




\title{
Sans naissance, pas de mariage ? Le procès en nullité du mariage de Louis XII et Jeanne de France (1498)
}

\author{
Stéphanie RICHARD \\ Université Paris-Sorbonne (Paris IV)
}

[...] $\mathrm{Vu}$ le procès mené et pendant devant nous, Philippe, du titre des saints Pierre et Marcellin de sainte Église romaine, cardinal de Luxembourg et évêque du Mans, et Louis d'Albi et Fernand de Ceuta, évêques, juges délégués en cette partie par notre saint père et seigneur en Christ Alexandre, pape sixième par la providence divine, entre le très chrétien Louis XII, roi des Francs, demandeur dans la cause de nullité de mariage, d'une part, et la très illustre dame Jeanne de France, défenderesse, comme parties, d'autre part [...] par notre sentence définitive [...] prononçons le mariage contracté entre les parties $[\ldots]$ ne pas avoir tenu ni ne tenir, mais avoir été et être nul et de nulle force, [...] et ne pas empêcher ledit seigneur demandeur d'être capable de contracter mariage ayant effet devant Dieu avec une autre $[\ldots]^{1}$

${ }^{1}$ Traduction du dictum de la sentence de nullité du mariage de Louis XII et de Jeanne de France: " [...] Viso processu moto et pendente coram nobis, Philippo, tituli sanctorum Petri et Marcellini sancte romane ecclesie presbitero cardinali de Lucemburgo, Cenomanensi, ac Ludovico Albiensi et Fernando Septensi, episcopis, judicibus a sanctissimo in Christo patre et domino, domino Alexandro, divina providencia pape sexto in hac parte delegatis, inter christianissimum Ludovicum duodecimum, Francorum Regem, in causa nullitatis matrimonii actorem, ex una, et illustrissimam dominam Johannam de Francia, ream, partibus, ex altera [...] per hanc nostram sententiam diffinitivam [...] pronunciamus matrimonium inter ipsas partes contractum [...] non tenuisse aut tenere, sed fuisse et esse nullum nuliusque momenti, [...] nec obstare quominus cum alia matrimonium efficax in Domino contrahere valeat prelibatus dominus actor » (c'est nous qui traduisons le latin, ici et 
C'est par cette sentence que le 17 décembre 1498, les juges chargés de statuer sur la validité du mariage de Louis XII et de Jeanne de France closent le procès opposant le roi à son épouse. Jeanne (1464-1505), deuxième fille du roi Louis XI, avait été promise quelques jours après sa naissance au jeune Louis II (1462-1515), fils du duc Charles d'Orléans (et futur Louis XII $)^{2}$. Effectivement célébré en $1476^{3}$, le mariage n'a rien eu d'heureux : Louis, détestant sa disgracieuse compagne, a vécu autant que possible hors de sa compagnie, bien que Jeanne se soit comportée en épouse dévouée. L'attitude de la princesse n'a pas empêché son mari d'engager une procédure judiciaire pour se dégager de son mariage, et ce dans les premières semaines de son règne $e^{4}$. Le déroulement de ce procès est plutôt bien connu car les pièces en ont été conservées ${ }^{5}$ et ont été retranscrites au XIX ${ }^{\mathrm{e}}$ siècle par René de Maulde La Clavière ${ }^{6}$.

A la fin de l'année 1498, les juges accèdent finalement à la requête de Louis XII en prononçant la nullité de son premier mariage. Par conséquent, vingt-deux ans après la célébration des noces, tous doivent désormais considérer que les liens conjugaux unissant le roi à la fille de Louis XI n'ont en réalité jamais existé. Toutefois, la sentence nous en apprend davantage. Conformément au droit canon, en effet, les juges n'ont

dans l'ensemble de notre article). Ce texte est publié par René de Maulde La Clavière, dans Procédures politiques du règne de Louis XII, Paris, Imprimerie Nationale, coll. «Documents inédits de l'histoire de France», 1885, pièce 59 " Dictum de la sentence de divorce », cit. p. 941-943.

${ }^{2}$ Voir René de Maulde La Clavière, Jeanne de France, duchesse d'Orléans et de Berry (1464-1505) d'après des documents inédits, recueillis par l'auteur avec la collaboration de MM. Sorin et de la Guère, Paris, Champion, 1883, p. 12.

${ }^{3}$ Ibid., p. 92.

${ }^{4}$ Louis II d'Orléans devient le roi Louis XII à la mort de Charles VIII le 7 avril 1498. Le procès s'ouvre le 10 août 1498 .

5 Il en existe trois copies authentiques: Paris, BnF, lat. 5973; Nantes, Archives Départementales de Loire-Atlantique, E 15 ; Albi, Bibliothèque municipale, ms. 31 ( ${ }^{\circ}$ CGM 78).

${ }^{6}$ René de Maulde La Clavière, Procédures politiques..., op. cit., p. 787-1132. 
pas indiqué sur quel(s) critère(s) ils ont fondé leur décision ${ }^{7}$. Louis XII, quant à lui, avait demandé la séparation en invoquant plusieurs empêchements dirimants de mariage ${ }^{8}$ : défaut d'âge ${ }^{9}$, consentement donné sous la menace, consanguinité et parenté spirituelle avec Jeanne de France, impuissance de la princesse enfin, la mettant dans l'incapacité de consommer le mariage et d'avoir des enfants. Or, seul Louis est autorisé explicitement à contracter une nouvelle union. Le tribunal semble ainsi reconnaître implicitement que Jeanne n'est pas apte à conclure une nouvelle alliance ; cela revient en définitive à désigner l'incapacité sexuelle de la fille de Louis XI comme argument décisif dans le procès, car c'est le seul empêchement qui affecte la princesse uniquement et qui est susceptible d'entraver l'aptitude de Jeanne à se remarier ${ }^{10}$.

${ }^{7}$ Le droit canon exige seulement des juges leur certitude morale sur l'affaire pour qu'ils puissent se prononcer. Voir Guillaume Durand, Speculum iudiciale; illustratum et repurgatum a Giovanni Andrea et Baldo degli Ubaldi [1561], Aalen, Scientia Verlag, 1975, livre II, partie III, rubrique De Sententia et de is quae ipsam sequuntur, $\S 5, \mathrm{n}^{\circ} 13$ et dans le Décret de Gratien II, Cause II, q. 1, c. 1. Notre édition de référence sera celle d'Emil Friedberg : Corpus iuris canonici. Pars prior, decretum magistri Gratiani [1879], éd. Emil Friedberg, Graz, Akademische Druck Verlagsanstalt, 1959.

${ }^{8}$ C'est-à-dire des circonstances ou des propriétés interdisant de contracter valablement une alliance. Le droit canon fait la distinction entre les empêchements dirimants, qui entraînent la nullité du mariage, et prohibitifs, sanctionnés en tant que faute mais qui ne mettent pas en cause le lien matrimonial (Adhémar Esmein, Le mariage en droit canonique [1891], Paris, Librairie du Recueil Sirey, 1929-1935 ( $2^{\mathrm{e}}$ édition mise à jour par Robert Génestal et Jean Dauvillier), t. I, p. 228-229).

${ }^{9}$ Pour pouvoir contracter mariage, il faut avoir atteint l'âge de la puberté, soit, selon le droit canon, 14 ans pour les garçons et 12 ans pour les filles (Adhémar Esmein, op. cit., t. I, p. 236). L'évocation du problème de l'âge s'explique par un certain flottement autour des dates de naissance et de célébration du mariage des deux conjoints.

${ }^{10}$ Signalons que pour Jean-François Drèze, les juges ne cautionnent pas jusqu'au bout cette logique puisqu'ils n'émettent pas de «vetitum Ecclesiae ad nuptias transire » à l'encontre de Jeanne de France (Jean-François Drèze, Raison d'Etat, raison de Dieu: Politique et mystique chez Jeanne de France, Paris, Beauchesne, 1991, p. 110). Toutefois, on notera que si le Corpus iuris canonici précise bien que les impuissants ne sont pas aptes au mariage et que le conjoint capable sexuellement peut se remarier, il n'est pas fait mention d'un veto spécifique à appliquer à l'époux empêché (voir Corpus iuris canonici, X, livre IV, titre 15, De frigidis et maleficiatis, et impotentia coeundi, c. 2 et c. 1 ; Liber extravagantium decretalium, dans Corpus 
Le procès engagé par le roi à l'encontre de Jeanne de France attire donc l'attention sur une séparation conjugale articulée autour de l'inaptitude à établir des relations sexuelles et, par suite, autour de l'impossibilité de concevoir des enfants. En d'autres termes, c'est un cas de «non naissance » et ses conséquences que la nullité du premier mariage de Louis XII invite à étudier. Dans cette perspective, la situation particulière de la procédure judiciaire offre un angle d'approche original. Les procès sont en effet à la fois un cadre d'énonciation des règles - ici ecclésiastiques - et un moment où les acteurs affichent leurs motivations en les modulant en fonction de ce que le tribunal, voire plus largement la société, jugent acceptable. Les règlements judiciaires des litiges sont donc l'occasion d'une confrontation entre les normes collectives et les intérêts particuliers. Ils sont aussi le cadre de la mise en pratique des règles, et donc de l'éventuelle inflexion de celles-ci ${ }^{11}$.

iuris canonici. Pars secunda, decretalium collectiones [1881], éd. Emil Friedberg, Graz, Akademische Druck Verlagsanstalt, 1959). Une interdiction expresse de ce type est en revanche évoquée par Hostiensis à l'encontre du mari atteint de frigidité (voir Hostiensis - Henri de Suze -, Summa Aurea, Venezia, J. Vitali, 1574, livre IV, rubrique De Frigid. \& Maleficiatis, \& de Impotentia coeundi, $\left.\mathrm{n}^{\circ} 10\right)$; mais si l'on en rencontre 1'application dans les registres de l'officialité de Cerisy (Gustave Dupont, Le registre de l'officialité de Cerisy 1314-1457, Caen, F. Le Blanc-Hardel, 1880, $\mathrm{n}^{\circ} 54 \mathrm{a}$ ), ce n'est pas le cas dans les registres des sentences de Bruxelles (Liber Sentenciarum van de Officialiteit van Brussel, éd. Cyriel Vleeschouwers et Monique Van Melkebeek, Bruxelles, Ministerie van Justitie, coll. «Recueil de l'ancienne jurisprudence de la Belgique $», 7^{\mathrm{e}}$ série, 1982, vol. 1, sentence 141). Le comportement des juges en 1498 ne paraît donc pas irrégulier ni inhabituel.

${ }^{11}$ L'étude de la jurisprudence médiévale en matière matrimoniale a d'ailleurs donné lieu à plusieurs publications depuis les années 1950. On peut par exemple mentionner, sans prétendre à l'exhaustivité, les travaux suivants : Anne Lefebvre-Teillard, Les officialités à la veille du Concile de Trente, Paris, Librairie générale de droit et de jurisprudence, coll. «Bibliothèque d'histoire du droit et droit romain », 19, 1973 ; Richard H. Helmholz, Marriage Litigation in Medieval England, Cambridge/London, Cambridge University Press, coll. "Cambridge studies in English legal history », 1974; Monique Vleeschouwers-Van Melkebeek, "Incestuous Marriages: Formal Rules and Social Practice in the Southern Burgundian Netherlands ", dans Love, Marriage and Family Ties in the Later Middle Ages, dir. Isabel Davis, Miriam Müller, Sarah Rees Jones, Brepols, Turnhout, coll. «International medieval research», 2003, p. 77-95; Charles Donahue Jr., Law, Marriage and Society in the Later Middle Ages : Arguments about Marriage in Five 
Il s'agira donc de considérer le procès de nullité de 1498 comme un moment de cristallisation des conceptions et des pratiques ecclésiastiques et laïques relatives aux liens entre naissance et mariage, dans le royaume de France à la fin du Moyen Âge. C'est d'abord le point de vue de la noblesse qui sera mis en valeur. Il faudra ensuite examiner les liens que l’Église établit entre mariage et filiation, à fois sous un angle théorique, mais également sur le plan pratique à travers l'application des règles au cas du mariage de Louis XII. Nous pourrons enfin examiner les réactions que la sentence de nullité a provoquées afin d'étudier la façon dont la population percevait cet enjeu de la naissance.

\section{Le point de vue de la noblesse : mariage et double enjeu dynastique}

L'idée que la naissance d'un héritier soit une préoccupation fondamentale pour les nobles à l'époque médiévale n'a rien de nouveau. Dans le cas qui nous intéresse, cependant, ce problème se présente sous la forme d'un double enjeu.

\section{La perpétuation de la lignée}

Au cours du procès, l'incapacité de Jeanne de France à engendrer des enfants est au cœur des arguments avancés par le roi pour faire valoir la nullité de l'union. Ainsi, dans sa bulle instituant le tribunal, Alexandre VI rappelle les réclamations qui lui ont été adressées par Louis XII : entre

Courts, Cambridge/New York/Melbourne, Cambridge University Press, 2007 ; Martine Charageat, La délinquance matrimoniale: couples en conflit et justice en Aragon au Moyen Âge (XV ${ }^{e}-X V I^{e}$ siècle), Paris, Publications de la Sorbonne, coll. « Histoire ancienne et médiévale », 106, 2011. 
autres raisons, le roi a fait valoir que Jeanne est « impuissante à concevoir une descendance ${ }^{12} »$.

Une fois les procédures entamées, la terminologie employée par le procureur Antoine de l'Estang, qui représente le roi, est plus complète. On lit par exemple dans ses conclusions préliminaires que

Jeanne de France fut par nature, comme elle est de présent, imparfaite, viciée de corps et soumise à un maléfice, inapte à l'homme, de sorte qu'elle n'eût pu ou ne pût concevoir, recevoir la semence virile selon la conformité de la nature, ni par aucun moyen être connue naturellement par un homme dans les enceintes des parties sexuelles ${ }^{13}$.

Comme on le voit, l'enjeu de la descendance côtoie cette-fois-ci les descriptions plus techniques de l'impuissance sexuelle ${ }^{14}$; toutefois la préoccupation dynastique reste présente ${ }^{15}$. Remarquons que c'est alors le besoin d'une postérité en général qui est mis en exergue : à la fin du Moyen Âge, pourtant, le principe de succession masculine à la Couronne de France est bien établi ${ }^{16}$. En fait, les modulations dans le discours

12 «ad concipiendum prolem impotens» (René de Maulde La Clavière, Procédures politiques..., op. cit., pièce 2 «Bulle d'Alexandre VI instituant le tribunal», cit. p. 813).

${ }^{13}$ «Johanna de Francia a natura fuit, prout est de presenti, imperfecta, corpore viciata et maleficiata, non apta viro, sic quod non potuisset aut posset concipere, semen virile secundum congruenciam nature recipere, ymo neque a viro intra claustra pudoris naturaliter cognosci $\gg$ (Paris, BnF, lat. 5973, $\mathrm{f}^{0} 7 \mathrm{r}^{\circ}$. Nous avons recours ici à ce manuscrit plutôt qu'à la version imprimée des pièces car René de Maulde n'a pas toujours reproduit les détails sexuels les plus précis dans son ouvrage, Procédures politiques..., op. cit.).

${ }^{14}$ L'association des deux aspects - incapacité à concevoir une descendance et données médicales sur l'impuissance physique - se perpétue ensuite dans les actes émanant $\mathrm{du}$ procureur du roi. Voir par exemple les articles d'Antoine de l'Estang pour l'interrogatoire des témoins (René de Maulde La Clavière, Procédures politiques..., op. cit., p. 958). Sur l'argument du maléfice, voir infra, en particulier note 36.

${ }^{15}$ Par ailleurs, dans le même acte, le procureur du roi insiste à nouveau sur ce point en précisant que Louis XII songe à la succession de son règne et à la nécessité de ne pas manquer de descendance (René de Maulde La Clavière, Procédures politiques..., op. cit., pièce 4 « Conclusions du procureur du roi », p. 822).

${ }^{16}$ Il en est d'ailleurs de même pour les apanages comme le duché d'Orléans. Si les femmes se voient très régulièrement reconnaître le droit de succéder aux fiefs depuis 
d'Antoine de l'Estang - description de l'impuissance, terminologie vague sur la descendance - sont des réponses aux impératifs du droit canonique, que nous aurons l'occasion d'évoquer par la suite.

L'enjeu dynastique est un problème partagé par les nobles en général : il est d'ailleurs perçu alors même que Louis n'est encore que duc d'Orléans. Le témoin Pierre Dupuy évoque en effet lors du procès les angoisses de Marie de Clèves au sujet du mariage de son fils avec la fille de Louis XI : la duchesse s'attriste par avance car elle pense que Louis ne s'occupera pas de sa femme, sous-entendant de la sorte qu'aucun héritier ne viendra à naître de cette union; par conséquent, la maison d'Orléans sera perdue ${ }^{17}$. On retrouve ici l'un des objectifs des stratégies familiales de la noblesse tels qu'ils ont été définis par Michel Nassiet : le mariage doit permettre la reproduction démographique de la lignée - ce que l'union avec Jeanne de France ne permettra pas, selon Marie de Clèves, de réaliser. Dans un deuxième temps, l'accession au trône de Louis XII viendra modifier l'enjeu dynastique en ajoutant à la reproduction physique la reproduction de l'influence publique par la transmission de la charge royale d'un père à son fils ${ }^{18}$.

le $\mathrm{X}^{\mathrm{e}}$ siècle (notamment en l'absence d'héritier mâle), ce n'est pas le cas pour les apanages, transmis depuis 1314 en ligne masculine uniquement (la règle étant confirmée en 1367 à propos de la succession de Philippe d'Orléans); quant à l'exclusion des femmes de la succession royale, elle s'est mise en place progressivement à partir des crises de succession de 1316 et 1328 et a été formalisée dans le droit par les ordonnances royales de 1374 et 1407.

${ }^{17}$ René de Maulde La Clavière, Procédures politiques..., op. cit., « Déposition de Pierre Dupuy, sire de Vatan », p. 985. On peut recouper ce témoignage avec la déposition du général des finances Michel Gaillard (René de Maulde La Clavière, Procédures politiques..., op. cit., p. 1025): «et pluries domina ducissa Aurelianensis dixit loquenti quod non curaret quam habuisset in uxorem, dummodo fuisset apta ad prolem suscipiendam » (" et à plusieurs reprises la dame duchesse d'Orléans dit au témoin qu'elle ne se soucierait pas de qui il avait eu comme épouse, pourvu qu'elle eût été apte à avoir des enfants »); cette fois, c'est l'incapacité physique de Jeanne à concevoir une descendance qui est directement pointée par la duchesse.

${ }^{18}$ Sur les objectifs de reproduction démographique et de reproduction de l'influence publique dans les stratégies familiales de la noblesse, voir Michel Nassiet, Parenté, 
Le problème de la naissance est donc central dans ce procès. Il faut cependant nuancer son importance : la demande en nullité du roi s'est articulée autour de plusieurs empêchements et non pas de la seule incapacité de Jeanne. Dans ce cadre, la question du consentement vicié de Louis XII, que ce dernier affirme avoir donné à contrecœur et sous la menace de son beau-père ${ }^{19}$, est également très débattue. Mais l'existence de cet empêchement apparaît liée au problème de la naissance: si Louis encore duc refuse de prendre Jeanne pour épouse, c'est semble-t-il parce qu'elle est réputée stérile ${ }^{20}$. Par conséquent, derrière la multiplicité affichée des motifs canoniques de nullité, l'incapacité de Jeanne de France à concevoir serait le véritable enjeu déterminant Louis XII à engager ce procès. Malgré tout, signalons que la motivation successorale n'est pas la seule à inspirer le comportement du roi. Bien que cela ne soit pas évoqué au cours de la procédure, si Louis XII redevenait célibataire en 1498, il serait alors libre d'épouser la veuve de Charles VIII, Anne de Bretagne, et donc d'arrimer à la France le duché dont elle est héritière ${ }^{21}$.

\section{L'extinction d'une famille}

Le problème dynastique peut également apparaître comme un contreenjeu dans le procès de 1498. À plusieurs reprises, en effet, le roi est dit avoir été contraint par Louis XI de prendre Jeanne de France pour épouse. Dès la naissance de sa fille, l' « universelle aragne » semble avoir résolu de

noblesse et États dynastiques, $X V^{e}-X V I^{e}$ siècles, Paris, Éditions de l'EHESS, coll. « Recherche d'histoire et de sciences sociales », 90, 2000, p. 30.

19 René de Maulde La Clavière, Procédures politiques..., op. cit., pièce 2 «Bulle d'Alexandre VI instituant le tribunal », p. 813.

${ }^{20}$ Les remarques régulières que Louis fait à ses serviteurs avant de devenir roi vont dans ce sens, car il affirme qu'il préfèrerait être marié à une fille pauvre mais capable d'avoir des enfants; voir par exemple ibid., "Déposition du secrétaire Jean Cotereau », p. 1048.

${ }^{21}$ C'est d'ailleurs ce qu'il s'empresse de faire dès le 8 janvier 1499. 
procéder à l'alliance de Jeanne et du futur duc d'Orléans ${ }^{22}$. Ensuite, c'est sous la menace que le vieux roi aurait obtenu le renouvellement des promesses de mariage entre Jeanne et Louis en 1473 puis serait parvenu à faire célébrer leurs noces en $1476^{23}$. Or, quand Jeanne vient à peine de naître, son père ne cherche probablement qu'à resserrer les liens entre la famille d'Orléans et la maison royale. En revanche, en 1473, Charles, futur Charles VIII, est né. Pour certains historiens, Louis XI vise alors à s'assurer du soutien du duc d'Orléans envers son fils en le mariant à l'une de ses filles - mais pas à l'aînée, de crainte que cela ne lui donne trop d'ascendant sur Charles en cas de tutelle $\mathrm{e}^{24}$. Quelques-uns, comme Abel Destefanis, ajoutent que le roi, sachant sa fille incapable d'enfanter, l'aurait donnée en mariage à Louis d'Orléans dans le but précis d'empêcher ce dernier d'avoir une descendance. Ainsi l'apanage, à défaut d'héritier mâle, serait retourné à la Couronne de France ${ }^{25}$.

Ce machiavélisme de Louis XI est évoqué dans plusieurs dépositions favorables à Louis XII. Le sire de Rabaudanges, ancien écuyer de Marie de Clèves, rapporte par exemple les entretiens de la duchesse avec certains de ses serviteurs, au cours desquels elle aurait affirmé savoir que Louis XI désirait éteindre la maison d'Orléans par un mariage stérile ${ }^{26}$. Surtout, une

${ }^{22}$ Voir note 2.

${ }^{23}$ Vers 1473, le roi aurait menacé Marie de Clèves de faire enfermer son fils dans une abbaye, voire de le faire mourir, s'il ne consentait pas à épouser Jeanne (René de Maulde La Clavière, Procédures politiques..., op. cit., «Déposition de Gilles des Ormes ancien grand maître des eaux et forêts d'Orléans », p. 983). Le témoignage de François de Brilhac, évêque d'Orléans, laisse supposer qu'en 1476 Louis XII n'a accepté de célébrer cette union que sous la contrainte (ibid., p. 1018).

24 Cette logique est d'autant plus plausible que Louis d'Orléans est le premier prétendant à la Couronne si la lignée masculine directe de Louis XI vient à s'éteindre : il est donc intéressant pour Louis XI d'en faire un allié. Voir René de Maulde La Clavière, Jeanne de France ..., op. cit., p. 63.

${ }^{25}$ Abel Destefanis, Louis XII et Jeanne de France : étude historique et juridique sur une cause de nullité de mariage à la fin $d u X V^{e}$ siècle, Avignon, Presses universelles, p. 39-40.

${ }^{26}$ René de Maulde La Clavière, Procédures politiques..., op. cit., p. 1058 : « et quod ipsa cognoscebat quod ipse Rex Ludovicus undecimus volebat et intendebat 
lettre du père de Jeanne lui-même, adressée au comte de Dammartin en 1473, permet d'appuyer l'hypothèse d'une stratégie méditée par Louis XI à l'encontre de son futur gendre. On y lit :

je me suis délibéré de faire le mariage de ma petite fille Jehanne et du petit duc d'Orléans, pour ce qu'il me semble que les enffans qu'ilz auront ensemble ne leur cousteront guere à nourrir ${ }^{27}$

La procréation peut donc prendre la forme d'un double enjeu dans la noblesse : d'abord, il peut s'agir de rechercher la naissance afin de défendre la perpétuation de sa propre lignée; mais l'incapacité à concevoir peut aussi être utilisée à des fins politiques contre des lignées concurrentes. Le cas qui nous est présenté est bien sûr singulier, puisque Louis XI, en tant que roi, possédait les moyens de coercition lui permettant de mettre en œuvre sa volonté; cela n'en est pas moins révélateur d'une attitude instrumentaliste vis-à-vis de la naissance.

\section{L’Église, le mariage et la descendance royale}

\section{La doctrine et le droit canon}

Le droit canon ne reconnaît pas nécessairement la nullité d'un mariage lorsque les conjoints sont incapables d'avoir des enfants ensemble. L'Église fait en effet une distinction entre la stérilité (impotentia generandi), le maléfice (maleficium, sortilège jeté par un tiers et qui empêche l'union sexuelle entre les conjoints), et l'impuissance sexuelle

destruere et sopire domum Aurelianensem per husjusmodi matrimonium, ex quo remaneret Rex modernus cum ipsa domina Johanna sine prole» (« et qu'elle savait que le Roi Louis XI voulait et s'appliquait à détruire et éteindre la maison d'Orléans par ce mariage, par lequel le Roi actuel resterait avec cette dame Jeanne sans descendance $\gg)$.

27 Ibid., pièce 49 «Production d'une lettre de Louis XI au comte de Dammartin et enquête sur son authenticité », p. 915-916. L'authenticité de cette lettre a été mise en doute ; René de Maulde et Abel Destefanis penchent néanmoins en faveur de l'idée que Louis XI l'avait effectivement fait rédiger puisqu'en 1488 Charles VIII tente de la récupérer auprès du comte de Dammartin (voir René de Maulde La Clavière, Jeanne de France ..., op. cit., p. 297-298 et Abel Destefanis, op. cit., p. 163-164). 
(impotentia coeundi, qui regroupe l'absence ou la déformation des organes sexuels et la frigidité). Sur ces trois cas, seuls l'impuissance - lorsqu'elle est perpétuelle et antérieure au mariage - et le maléfice permettent la séparation des époux ; la stérilité, quant à elle, n'est pas considérée comme un motif de nullitée ${ }^{28}$. L'explication tient en partie à des considérations religieuses plus générales sur les fins du mariage. Depuis saint Augustin, en effet, on lui reconnaît trois « biens » (bona) : la génération (proles), dont la priorité est incontestée ${ }^{29}$; la fidélité (fides), soit l'assistance mutuelle consistant à aider le conjoint à porter sa propre faiblesse d'incontinence sexuelle ; le sacrement (sacramentum), l'union de l'homme et de la femme étant le signe de l'union indissoluble du Christ et de son Église ${ }^{30}$.Or, l'impuissance et le maleficium, qui empêchent tout acte sexuel, privent les conjoints des bénéfices de toutes les fins du mariage : celui-ci peut donc être considéré comme nul. En revanche, la seule infertilité ne rend impossible que la génération physique et maintient donc la validité du mariage ${ }^{31}$. Par conséquent, dans une cause de nullité ayant trait à ces questions, ce n'est pas la naissance qui est le cœur du sujet. Toutefois, cet enjeu ne disparaît pas totalement : en tant qu'un des biens du mariage, il reste probablement un horizon dans l'esprit des membres du tribunal ${ }^{32}$ et

${ }^{28}$ Adhémar Esmein, op. cit., t. I, p. 270-271 et 276-278.

${ }^{29}$ Néanmoins, l'objectif premier de génération chez saint Augustin ne s'arrête pas à la procréation physique : pour l'évêque d'Hippone, il s'agit également de s'occuper de l'éducation morale et spirituelle des enfants dans la religion chrétienne, dans un contexte où l'engendrement des enfants n'est plus aussi nécessaire que dans les temps anciens (voir Émile Schmitt, Le mariage chrétien dans l'œuvre de Saint Augustin: une théologie baptismale de la vie conjugale, Paris, Études augustiniennes, coll. «Études augustiniennes, série Antiquité », 97, 1983, p. 265266).

${ }^{30}$ Voir saint Augustin, Le bien du mariage dans Le bien du mariage-La virginité consacrée, trad. Gustave Combès, Paris, Institut d'études augustiniennes, coll. Nouvelle bibliothèque augustinienne, p. 21-76.

${ }^{31}$ Voir Adhémar Esmein, op. cit., t. I, p. 278.

${ }^{32}$ On notera d'ailleurs que plusieurs des décrétales du Corpus iuris canonici traitant de la séparation des époux pour impuissance évoquent le désir du conjoint capable 
l'on peut également penser que ces hommes d'Église étaient en même temps bien conscients de l'urgence dynastique qui préoccupait l'aristocratie $\mathrm{e}^{33}$. Mais dans une procédure judiciaire, c'est bien le problème de l'incapacité physique à avoir des relations sexuelles qui concentre l'attention des juges. Cela est d'ailleurs manifeste dans la bulle par laquelle Alexandre VI instaure le tribunal : si les arguments de Louis XII, tels qu'ils sont rappelés par le pape, évoquent l'impossibilité pour Jeanne de France de concevoir des enfants, Alexandre VI se place directement sur le plan «médical» en intimant l'ordre aux juges de vérifier si Jeanne est mal conformée ou soumise à un maléfice de sorte que le roi ne puisse la connaitre charnellement ${ }^{34}$. On comprend donc pourquoi le discours d'Antoine de l'Estang s'infléchit dans un sens plus technique face aux juges. Ce cadre permet également de saisir pourquoi le roi et son procureur restent sur un plan général lorsqu'ils parlent de «descendance » : l’Église reconnaît la procréation en général comme l'un des buts du mariage sans qu'il s'agisse d'une procréation sélective en fonction du sexe de l'enfant. L'argumentation a donc été adaptée au contexte de son énonciation.

sexuellement d'être parent; voir par exemple X, livre IV, titre 15, De frigidis et maleficiatis, et impotentia coeundi, c. 6 pour un homme et c. 7 pour une femme.

33 A titre de comparaison, on peut souligner qu'après déclaration de la nullité du mariage de Charles IV en 1322, le pape Jean XXII a invité le roi à prendre rapidement une nouvelle épouse robuste pour assurer sa descendance. Voir les Lettres secrètes et curiales (relatives à la France) de Jean XXII, $\mathrm{n}^{\circ} 001436$, en date $\mathrm{du} 24$ juin 1322 (disponible en ligne sur la base de Brepolis, Ut per litteras apostolicas, http://apps.brepolis.net.janus.biu.sorbonne.fr/litpa/Pontificates.aspx, base consultée le 30 octobre 2013).

34 René de Maulde La Clavière, Procédures politiques..., op. cit., pièce 2 «Bulle d'Alexandre VI instituant le tribunal », cit. p. 814 : «quodque dicta Johanna adeo maleficiata et impotens existat quod idem Ludovicus, modernus Rex, eam interim carnaliter, intra claustra pudoris, cognoscere nullathenus potuit neque potest » (« et que la dite Jeanne soit si soumise à un maléfice et impuissante que le même Louis, Roi actuel, dans l'intervalle n'a pu ni ne peut la connaître charnellement en aucune façon dans l'enceinte des parties sexuelles »). 


\section{L'application des règles au procès de 1498}

L'essentiel des débats lors du procès se concentre autour du vice de consentement d'une part, et d'autre part de l'impuissance de Jeanne en raison de la déformation de ses organes sexuels $\left(\right.$ arctatio $\left.^{35}\right)$ et d'un maleficium $^{36}$. Ces deux empêchements sont liés, puisque le droit canon établit que la crainte ayant vicié le consentement d'un conjoint est purgée par la cohabitation spontanée des époux et surtout par l'union charnelle ${ }^{37}$ qui, chez l'homme, est toujours présumée volontaire ${ }^{38}$. Par conséquent, la consommation ou non du mariage est l'un des nœuds du procès. Le roi

${ }^{35}$ Dans le droit canon, l'impuissance féminine n'est reconnue comme empêchement dirimant qu'à partir des décrétales de la fin du XII ${ }^{\mathrm{e}}$ siècle. Il est alors généralement admis qu'elle peut prendre deux formes : la clausura (absence des organes génitaux ou leur fermeture) et l'arctatio (leur inaptitude à l'acte sexuel). Voir Adhémar Esmein, op. cit., t. I, p. 276.

${ }^{36}$ Voir note 13 . L'argument du maléfice, avancé par le procureur du roi, a été très peu débattu (l'essentiel des discussions portant sur l'impuissance physique) mais il a posé quelques difficultés au cours du procès. Pour éviter à Jeanne de France de se soumettre à un examen médical, la défense de la princesse a allégué que ce type de sortilège ne peut affecter une personne déjà impuissante par nature et que par conséquent Jeanne devait être considérée comme normalement constituée (René de Maulde La Clavière, Procédures politiques..., pièce 32 «Conclusions de Jeanne de France contre l'examen corporel », p. 860). En retour, Antoine de l'Estang a argué $\mathrm{du}$ fait que Jeanne n'était pas seulement présentée comme maleficiata mais également comme impuissante et que cette impuissance physique devait être constatée par des personnes expertes (ibid., pièce 33 « Réplique du procureur du roi aux fins de l'examen corporel », p. 863-864). D'après Abel Destefanis, le procureur du roi avait simplement à l'origine utilisé un terme impropre en parlant de maléfice car ce type de sortilège ne peut affecter que les hommes (Abel Destefanis, op. cit., p. 166).

${ }^{37}$ Voir le commentaire du Panormitain sur Corpus iuris canonici, X, livre IV, titre 1, De sponsalibus et matrimoniis, c. $28, \mathrm{n}^{0} 7$. Notre édition de référence est la suivante : Niccolò Tedeschi, Nicolai Tudeschii Catinensis Siculi Abbatis Panormitani, Commentaria in Quartum et Quintum Librum Decretalium, Venetiis, Apud Iuntas [1588] / Cambridge (Mass.), Omnisys, 1990 (reproduction sur microfilm).

${ }^{38}$ On trouve cet argument dans la défense de Jeanne de France (René de Maulde La Clavière, Procédures politiques..., op. cit., pièce 41, p. 873). Effectivement, d'après Hostiensis, l'homme - contrairement à la femme - ne peut alléguer avoir été contraint à l'acte sexuel et donc le mariage tient, quoiqu'il y ait eu originellement vice de consentement de la part du mari, si l'échange des verba de praesenti a été suivi de l'union charnelle (Henrici de Segusio Cardinalis Hostiensis, In quartum decretalium librum Commentaria, Venetiis, apud Iuntas, 1581, commentaire sur Corpus iuris canonici, X, livre IV, titre 1 De sponsalibus et matrimoniis, c. 30). 
affirme ainsi qu'il n'a jamais pu connaître charnellement la princesse $^{39}$; Jeanne, de son côté, déclare avoir eu à plusieurs reprises des relations sexuelles non forcées avec son mari ${ }^{40}$.

La difficulté réside dans le fait que la princesse, faisant valoir sa pudeur naturelle et son ascendance royale ${ }^{41}$, tente d'éviter l'examen médical habituellement utilisé dans ce type de procédure pour apporter la preuve de l'impuissance physique ${ }^{42}$. Elle finit par refuser définitivement la visite corporelle et Jeanne défère alors à Louis XII le serment par lequel le roi doit confirmer que les positions qu'il soutient sont conformes à la vérité $^{43}$. Le roi jurant solennellement, entre autres, n'avoir jamais pu

${ }^{39}$ Et ce, bien qu'il ait été contraint de faire quelques tentatives; voir René de Maulde La Clavière, Procédures politiques..., op. cit., pièce 42 «Réplique du procureur du roi », p. 891. On remarquera que l'argumentation du roi ne précise pas clairement que l'impuissance sexuelle de Jeanne est incurable et donc perpétuelle, alors qu'elle laisse bien supposer qu'elle est antérieure au mariage (voir note 13).

40 Ibid., pièce 5, "Réplique sommaire de Jeanne de France », p. 825 ; pièce 41 «Défense présentée au nom de Jeanne de France », p. 875-876 et 878-880. Il est d'ailleurs à noter que la défense de Jeanne s'articule davantage autour de sa capacité sexuelle et peu autour de son aptitude à procréer, quoique cette dimension figure dans son argumentation (voir ibid., p. 883).

41 Ibid., pièce 32 : "Conclusions de Jeanne de France contre l'examen corporel », p. 861.

${ }^{42}$ Lorsque la cause de l'impuissance est matériellement constatable, comme en cas d'arctatio, le droit canon prescrit en effet le recours à l'expertise d'un groupe de médecins et de matrones (voir au Corpus iuris canonici, op. cit., X, livre IV, titre 15, De frigidis et maleficiatis, et impotentia coeundi, c. 6). En cas de maléfice, c'est normalement l'épreuve de la cohabitation triennale (délai de trois ans pendant lequel les époux doivent habiter ensemble en essayant de consommer le mariage) qui est imposée, accompagnée d'un serment avec sept cojurants (ibid., c. 7) ; toutefois, en pratique, le début de cette épreuve est fixé au commencement de la vie conjugale (voir Adhémar Esmein, op. cit., t. I, p. 292). Or les conjoints royaux sont ici mariés depuis vingt-deux ans.

${ }^{43}$ René de Maulde La Clavière, Procédures politiques..., op. cit., pièce 50 « Délation de serment au roi », p. 919-921. Entre temps, les témoins de Jeanne de France et du roi, interrogés sur l'impuissance de la fille de Louis XI ou sur la consommation du mariage, se sont généralement limités à décrire les infirmités physiques extérieures de la princesse, bossue, ajoutant parfois qu'en raison de ces défauts il était clair qu'elle ne pouvait pas faire d'enfant (voir par exemple la déposition de Guillaume Chaumart, religieux de Fontevrault, ibid., p. 971); d'autres affirment que les deux conjoints ont passé certaines nuits dans le même lit sans pour autant savoir s'il y a eu acte sexuel (voir par exemple la déposition de Jean Vigneron, auditeur des comptes, ibid., p. 1016). Les dépositions de Salomon de Bombelles et Jean Bourgeois, 
consommer le mariage $^{44}$, il n'est pas étonnant que le procès s'achève sur une déclaration de nullité.

Le règlement de cette séparation, en définitive, a toujours laissé subsister un doute sur la capacité de Jeanne de France à donner naissance à un héritier: le roi s'est-il parjuré, ou bien la princesse a-t-elle refusé l'examen corporel parce qu'elle se savait mal conformée? Les deux versions n'ont eu de cesse d'avoir l'une et l'autre des partisans. A défaut de pouvoir statuer sur cette question, les historiens tendent à souligner la validité formelle de la procédure judiciaire ${ }^{45}$, tout en en attirant l'attention sur certains traits suspects tels que le remplacement de l'un des juges pendant le procès. On notera en dernier lieu que pour Michel Lhospice, l'attitude favorable du pape envers le roi de France en 1498 a fortement orienté l'issue du procès, créant une sorte de précédent dans l'idée de séparation des couples royaux pour «non naissance » d'un héritier ${ }^{46}$.

médecins, sont en revanche plus précises en rapportant, à partir des récits que leur en a faits Louis XII, comment le roi avait échoué dans ses tentatives d'avoir des relations sexuelles avec sa femme (Paris, $B n F$, lat. 5973, $\mathrm{f}^{0} 209 \mathrm{v}^{\mathrm{o}}$ et $\mathrm{f}^{0} 162 \mathrm{v}^{0}$ à $163 \mathrm{r}^{\circ}$ ). Enfin, Michel Gaillard se fonde sur les paroles de Marie de Clèves pour décrire les possibles malformations internes de la princesse, à savoir un vagin naturellement étroit, avec une rétractation d'un côté et un obstacle osseux (René de Maulde La Clavière, Procédures politiques..., op. cit., «Déposition de Michel Gaillard», p. 1026). Le droit canon recommandait néanmoins aux juges la prudence vis-à-vis des témoignages de visu et auditu lorsqu'il s'agit d'établir l'union sexuelle (voir le Corpus iuris canonici, livre II, titre 20, De testibus et attestationibus, c. 27).

44 René de Maulde La Clavière, Procédures politiques..., op. cit., pièce 55 « Interrogatoire et serment du roi », p. 933.

${ }^{45}$ Voir par exemple Michel Lhospice, Divorce et dynastie, Paris, Librairie générale de droit et de jurisprudence/R. Pichon et R. Durand-Auzias, coll. Bibliothèque d'histoire du droit et du droit romain 3, 1960. La question de la validité de la procédure reste cependant discutée. On notera que, dans ce cadre, la régularité du serment prêté par le roi a constitué un point débattu par les historiens; pourtant, si l'on suit les prescriptions de Panormitain, ce serment n'aurait pas dû être retenu comme moyen de preuve (Niccolò Tedeschi, Commentaria in Quartum et Quintum Librum Decretalium..., op. cit., sur X, livre II, titre 24, De jurejurando, c. $34, \mathrm{n}^{0} 7$ ). Il n'est pas certain que sans ce juramentum les juges aient eu les preuves nécessaires pour rendre leur sentence.

${ }^{46}$ Michel Lhospice, op. cit., p. 40. Cet avis n'est pas non plus partagé par tous : Abel Destefanis, quant à lui, préfère insister sur le fait que le pape s'est tenu à l'écart des procédures (Abel Destefanis, op. cit., p. 11). 
$\mathrm{Au}$ bout du compte, ce procès montre qu'en dépit de règles bien établies, l'application pratique du droit canonique au cas d'un couple demeuré sans enfant reste complexe. Les buts poursuivis par les différents acteurs (époux, juges) et les relations que ces acteurs établissent entre eux viennent infléchir, jusqu'à un point difficile à définir ici, la mise en œuvre des règles ecclésiastiques reliant mariage et génération.

\section{La réception de l'enjeu de la naissance}

\section{Le point de vue des chroniqueurs}

A première vue, la nécessité de la procréation d'un héritier était une idée bien comprise dans le royaume de France. Par exemple, Nicole Gilles, officier de Louis XII, se sert de cet argument pour justifier le démariage et le remariage de son maitre en affirmant que Louis XII «scavoit bien par l'oppinion des grans medecins et philosophes qu'il ne pourroit avoir lignée [de Jeanne de France] a la raison de ce qu'elle estoit contrefaicte ${ }^{47}{ }$. C'est donc bien l'incapacité de Jeanne à engendrer un héritier, plutôt que son impuissance à établir des relations sexuelles, qui est mise en avant. De façon générale, ce motif n'est jamais rapporté seul : pour évoquer cette affaire, les auteurs rappellent également communément le vice de consentement $^{48}$. Mais il est significatif que l'enjeu dynastique soit mis en valeur : cela montre qu'il était reconnu comme une justification valable pour rechercher la nullité du mariage.

D'ailleurs, c'est ce même objectif successoral qui est rappelé dans des chroniques plus neutres ou plus critiques sur le résultat du procès de nullité. Ainsi, le Loyal Serviteur, qui met en doute la légitimité de la séparation ( Si ce fut bien ou mal fait, Dieu est tout seul qui le

${ }^{47}$ Nicole Gilles, Les tres elegantes et copieuses annales..., Paris, J. Longis, 1536, cit. p. CXXVv'.

${ }^{48}$ On peut aussi noter que Nicole Gilles mentionne l'objectif de conserver la Bretagne par un nouveau mariage avec la duchesse Anne (ibid.). 
congnoist $\left.{ }^{49} »\right)$, prend quand même le temps de rappeler la possible stérilité de la princesse en question ${ }^{50}$.

Les différents auteurs retiennent donc de l'affaire la stérilité de Jeanne, son incapacité à donner naissance à un héritier, bien plus que le problème de l'impuissance physique tel qu'il est envisagé par l'Église. Puisqu'ils en font un argument en faveur de Louis XII, on peut en conclure que l'enjeu dynastique lié au mariage était une préoccupation reconnue au moins par les personnes susceptibles de lire ces textes - en d'autres termes, surtout des nobles, ce qui n'est finalement pas étonnant.

\section{Le point de vue du peuple}

Que sait-on des réactions des autres catégories de population à propos de la séparation du roi et de la fille de Louis XI ? Sur ce sujet, les historiens évoquent souvent le récit qu'en fait la Chronique de l'Annonciade; d'après ce document datant du milieu du XVI ${ }^{\mathrm{e}}$ siècle, le jour de la sentence à Amboise, le peuple de la ville ému du sort de Jeanne se met en colère et pointe du doigt les juges en s'écriant «Voila Caïphe! Voila Anne ! Voila Herodes et Pilate qui ont donné la sentence contre la saincte Dame, qu'elle n'est plus royne de France ${ }^{51} »$. Évidemment, la véracité des faits rapportés dans ce récit peut être mise en doute: la Chronique est une œuvre à la gloire de Jeanne de France et elle cherche à mettre en valeur toutes les injustices dont la princesse a pu souffrir.

${ }^{49}$ Jacques de Mailles, Histoire du Seigneur de Bayart, le chevalier sans paour et sans reprouche, composée par le Loyal Serviteur, Paris/Cambridge, Droz/W. Heffer and sons, 1927, cit. p 45.

${ }^{50}$ On lit que le roi « avoit espousé madame Jehanne de France [...] ; mais au moyen de ce qu'on tenoit que d'elle ne pourroit sortir lignée, et que par force l'avoit espousée, [...] la fist appeler en justice » (ibid.).

51 Chronique de l'Annonciade. Vies de la Bienheureuse Jeanne de France et du Bienheureux Gabriel-Maria, O.F.M., éd. Jean-François Bonnefoy, Paris, Éditions franciscaines, coll. « La France franciscaine », 1, 1937, cit. p. 81. 
Il semble toutefois que le résultat du procès de 1498 ait effectivement suscité quelques réactions critiques. La preuve en est surtout que Louis XII a pris la peine de proclamer à plusieurs reprises la régularité de la procédure et la validité de sa nouvelle union avec la veuve de Charles VIII : d'abord, dès le 26 décembre 1498, dans l'ordonnance par laquelle le roi fait Jeanne duchesse de Berry ${ }^{52}$; puis le 10 janvier 1499, dans une lettre adressée à ses gens des comptes, qui n'a pas d'autre objectif particulier que d'affirmer la légitimité de ses actions ${ }^{53}$.

L'existence de tels documents laisse supposer que certaines personnes dans la population avaient dû manifester au moins de la surprise, sinon du mécontentement, à l'annonce de la séparation du roi et de sa première épouse. Par conséquent, on peut déjà en conclure que la caution d'une procédure officielle n'était pas suffisante pour garantir la légitimité d'une décision aux yeux de tous les hommes du Moyen Âge; certains d'entre eux avaient donc l'idée que l'application des règles canoniques pouvait être infléchie en fonction du contexte, des acteurs en présence et de leurs motivations ${ }^{54}$. Mais que faut-il en déduire plus spécifiquement sur la façon dont étaient considérés les liens entre naissance et mariage dans la population? Si certains ont protesté, c'est qu'ils ont estimé que le mariage de Louis XII et de Jeanne était valable. Il est cependant difficile de

${ }^{52}$ Ordonnances des rois de France de la troisième race, vingt et unième volume : Les ordonnances rendues depuis le mois de mai 1497 jusqu'au mois de novembre 1514, éd. Jean-Marie Pardessus, Paris, Imprimerie nationale, 1849, p. 141-144.

${ }^{53}$ Voir Jean Bernier, Histoire de Blois contenant les antiquitez et singularitez du comté de Blois, les éloges de ses comtes et les vies des hommes illustres qui sont nez au païs blésois..., Paris, F. Muguet, 1682, p. xxxvi-xxxvii.

54 Une telle conception était peut-être facilitée par la souplesse relative du droit canonique en matière d'application des lois; d'après Charles Lefebvre, "pour le droit canonique en effet, la loi est une règle susceptible de n'être pas appliquée en certaines circonstances en raison de l'intervention soit du législateur, soit de certains de ses représentants, et parfois même sans elle » (Charles Lefebvre, «Les exceptions à la norme dans le domaine du droit matrimonial canonique ", Revue de droit canonique, 30, 1978, p. 30-43, cit. p. 31) ; cette souplesse justifie l'existence des dispenses matrimoniales par exemple. 
déterminer si ce mécontentement provient de l'idée que le roi avait en réalité consommé son mariage avec la princesse, ou bien d'un éventuel principe - qui serait alors encore plus strict en matière d'indissolubilité que les règles canoniques - selon lequel une union de plus de vingt ans serait à considérer comme un véritable mariage, impuissance ou non - et donc naissance ou non. Dans cette deuxième hypothèse, il y aurait un décalage entre les buts attribués au mariage par certaines personnes dans la population et les enjeux dynastiques liés aux stratégies matrimoniales dans la noblesse. Dans le premier cas en revanche, ce ne serait que le parjure du roi et le caractère douteux de la procédure qui seraient réprouvés; néanmoins, cette même situation sous-entendrait qu'une partie du peuple, sachant que le mariage a été consommé bien qu'il ait été jusqu'à présent stérile, considère cette union comme valide. Cela signifierait finalement que l'engendrement d'une filiation, question centrale dans le discours du roi, n'est pas le cœur du problème en matière de séparation conjugale pour ses sujets. On en revient donc au même décalage entre les objectifs successoraux que la noblesse attribue aux alliances matrimoniales et les buts envisagés par une partie du peuple pour ce sacrement.

En définitive, le procès en nullité de mariage de Louis XII et de Jeanne de France révèle toute la variété des conceptions liant mariage et naissance. Dans la noblesse, les stratégies matrimoniales se combinent avec l'objectif dynastique, et les unions apparaissent d'abord sans surprise comme des outils visant la reproduction de la lignée. En tant que tels les mariages stériles sont donc à désunir : sans naissance, par conséquent, il n'y a pas de mariage qui tienne. Mais, ce qui est plus étonnant, c'est que ces alliances sans enfant peuvent aussi servir à l'occasion d'instrument contre des lignées concurrentes. La séparation de 1498 montre donc la naissance sous l'angle de deux enjeux contradictoires. L'Église s'inscrit par 
contre en décalage avec les perspectives de la noblesse : la procréation n'étant pas le seul bien du mariage, la validité d'une union n'est normalement pas subordonnée à la génération d'un héritier. Certains sujets dans le royaume de France semblent d'ailleurs adopter une conception similaire - mais pas nécessairement identique - quant aux liens entre procréation et mariage. L'application des normes en matière de mariage et de naissance recèle cependant des ambiguïtés. Dans une certaine mesure, les règles apparaissent donc modulables en fonction des circonstances et des personnes.

Enfin, on peut signaler que Louis XII remarié à Anne de Bretagne a bien obtenu en partie ce qu'il recherchait, à savoir une descendance; mais seules deux filles ont survécu, si bien que la Couronne a finalement échoué à une branche collatérale en la personne de François d'Angoulême. Jeanne de France, quant à elle, ne s'est jamais remariée. Fondatrice de l'ordre de l'Annonciade, elle fit vœu de religion; certains n'ont pas manqué de souligner la ferveur particulière de la princesse envers la Vierge mère alors qu'elle-même avait été officiellement délaissée par son mari en raison de son incapacité à mettre au monde un enfant ${ }^{55}$, et ce dans une société qui assigne aux femmes la procréation pour fonction principale ${ }^{56}$.

55 Louis-Hector Chaudru de Raynal, Histoire du Berry: depuis les temps les plus anciens jusqu'en 1789, Bourges, Vermeil [1844] / Paris, Éditions F.E.R.N., 1972, t. 3, p. 219.

${ }^{56}$ Voir Anne Lefebvre-Teillard, « Exposé de synthèse », dans La femme au Moyen Âge. Colloque organisé par la Faculté de droit Jean-Monnet, 12 avril 1991, Paris, La Documentation française, «Collection des journées de la faculté de droit Jean Monnet », 2, 1992, p. 71-76, en particulier p. 71. 